\title{
Propuestas de uso funcional para potenciar el turismo en el Municipio Artemisa como vía de desarrollo local
}

\section{Proposals for functional use to enhance tourism in the Artemisa Municipality as a means of local development}

Gerardo Carlos Hernández Rodríguez. ${ }^{1}$

\begin{abstract}
.
The Artemisa Province is the result of the new political-administrative division approved by the National Assembly of Cuba in August 2010. Its Provincial Capital, Artemisa municipality, is economically speaking, rich in the agricultural sector (coffee and sugarcane fundamentally), also standing out the industrial sector. Tourism, has been evaluated by the Historian of the Province and based on statistics, is very poorly developed. After in-depth research based on methods such as: bibliography, analysis and synthesis, induction-deduction and also arduous field work, a set of resources and attractions could be listed that have a low level of current use but have a high potential to link them to a proposal for functional use to develop tourism in the municipality as a local development strategy. The research and the results obtained allowed to propose three tourist modalities to develop and design three activities related to these
\end{abstract}

Keywords: training, Artemis, municipality, induction-deduction.

\section{Resumen.}

La Provincia Artemisa es resultado de la nueva división político-administrativa aprobada por la Asamblea Nacional de Cuba en agosto del 2010. Su Capital Provincial, municipio Artemisa, es económicamente hablando, rico en el sector agrícola (café y caña de azúcar fundamentalmente) destacándose también el sector industrial. El Turismo, ha valoración del Historiador de la Provincia y en base a estadísticas, está muy poco desarrollado. Luego de una profunda labor de investigación basándonos en métodos como: el bibliográfico, análisis y síntesis, inducción-deducción y también un arduo trabajo de campo, se pudieron listar un

\footnotetext{
${ }^{1}$ Universidad de la Habana, La Habana, Cuba. gerard.chr97@gmail.com
} 
conjunto de recursos y atractivos que presentan un bajo nivel de uso actual, pero tienen un alto potencial para vincularlos a una propuesta de uso funcional para desarrollar el turismo en el municipio como una estrategia de desarrollo local. La investigación y los resultados obtenidos, permitieron proponer tres modalidades turísticas a desarrollar y diseñar tres actividades relacionadas con estas

Palabras clave: capacitación, Artemisa, municipio, inducción-deducción.

\section{Introducción.}

La Provincia Artemisa es resultado de la nueva división político-administrativa aprobada por la Asamblea Nacional de Cuba en agosto del 2010 y que comenzó a funcionar el 9 de enero de 2011. Está conformada por 11 municipios y una superficie total de $4003.24 \mathrm{~km}^{2}$, por su tamaño la 13va del país.

El territorio artemiseño es eminentemente agrícola debido a la fertilidad de sus suelos, cuenta también con bellos paisajes en la heredada Sierra del Rosario y atractivas playas en el litoral del municipio de Bauta. En materia de turismo la provincia presenta un bajo nivel de desarrollo debido a la baja explotación de áreas que pudieran representar un atractivo potencial para el turismo.

Su Capital Provincial, municipio Artemisa (objeto de estudio de esta investigación) es, económicamente hablando, rico en producciones agrícolas, destacándose el café, la piña y la caña de azúcar. En la actualidad está representada por las empresas, de ellas 5 nacionales y una local, 4 unidades presupuestadas ganaderas, Avícolas y Porcinas, contribuyendo entre todos al crecimiento de un 13,9 \% de la producción mercantil. El Turismo es un renglón, que ha valoración del Historiador de la Provincia (Daniel Suárez Rodríguez), no se encuentra ni siquiera implementado o desarrollado como estrategia de crecimiento económico.

El municipio artemisa tiene una historia y una cultura muy rica en relación a la historia y la cultura del país de manera general. Su antepasado y el de sus habitantes, han estado interrelacionados directamente con la formación de los pilares histórico-culturales del cubano. Por lo tanto, en pos de diseñar una propuesta turístico-recreativa que tenga como principal objetivo contribuir al desarrollo local, como vía fundamental de gestión municipal, se decidió realizar un estudio de análisis de recursos y atractivos que tengan amplia vinculación con la historia y la cultura del municipio y que permitan desarrollar actividades turísticas relacionadas con estas.

A partir de ello se determinó como problema de investigación, la vinculación de las herencias histórico-culturales del municipio Artemisa al desarrollo de la actividad turística como vía de gestión en pos al desarrollo local. El objetivo general es diseñar una 
propuesta de uso funcional que permitan desarrollar la actividad turística en el municipio Artemisa. Como objetivos específicos se plantearon los siguientes:

- Realizar un estudio de campo que permita un acercamiento a los principales recursos y atractivos del municipio.

- Inventariar los recursos encontrados.

- Realizar una evaluación general de los recursos inventariados.

- Vincular los principales recursos a modalidades turísticas potenciales.

\section{Métodos.}

Para el desarrollo de la investigación se utilizaron varios métodos que permitieron la recopilación, evaluación, análisis y comparación de información. Dentro de estos:

Método bibliográfico: Permitió conocer los elementos teóricos necesarios en la investigación a través de la revisión bibliográfica.

Análisis - Síntesis: Garantizó la selección de las teorías adecuadas para el tratamiento del objeto de estudio a partir de los resultados científico - prácticos más actualizados sobre la temática.

Inducción - Deducción: Facilitó el establecimiento de relaciones adecuadas y la realización del estudio partiendo de lo general a lo particular.

Observación científica: Permite la percepción directa del objeto de investigación para conocer la realidad. En nuestro trabajo, se implementaron varias tablas que sirvieron de guía para la evaluación general de los recursos inventariados:

- Tabla 1: Tabla para la evaluación del estado y conservación de los recursos. En esta tabla se pudo estimar el valor conservativo, uso actual y tendencial, uso potencial y limitaciones de los recursos.

- Tabla 2: Tabla para la determinación del carácter atrayente o disuasivo del recurso, incluye además las recomendaciones.

- Tabla 3: Tabla de definición del valor de los recursos. En esta tabla se pudo valorar la unicidad, intensidad y geodiversidad de cada recurso, estableciendo un promedio para cada uno de estos.

- Tabla 4: Tabla de valoración de uso funcional. Esta tabla permite evaluar los recursos en relación a las modalidades propuestas. 
- Tabla 5: Tabla de evaluación turística de los atractivos. Esta tabla permite la evaluación estética, funcional, conservacionista, higiénico-sanitaria y técnicoeconómica de los recursos.

Entrevista: Se utilizó para justificar y argumentar toda la información del trabajo referente a los valores culturales de cada atractivo. Entrevistado: Daniel Suárez Rodríguez (Historiador de la Provincia Artemisa)

La presente investigación es importante porque se implementaría de forma directa en el municipio Artemisa como estrategia para su desarrollo local y también serviría como guía para otras regiones que presenten un bajo nivel de desarrollo turístico.

\section{Resultados.}

Luego de ardua labor de investigación y trabajo de campo, se pudieron listar un conjunto de recursos y atractivos, así como servicios que pertenecerían a la infraestructura de apoyo. La infraestructura de apoyo estaría conformada por la infraestructura turística (entidades de alojamiento, restauración y ocio) y la infraestructura de equipamiento (servicios generales, comunicaciones, instalaciones culturales e infraestructura rural). Ya por último se listaron un total de 20 recursos que guardan una estrecha relación con la cultura y la historia del municipio, elementos alrededor de los cuales, girará nuestra propuesta final.

\section{Recursos y atractivos ( $R$ ) inventariados:}

R1: Cafetal Angerona

R2: Cafetal Buena Vista

R3: Trocha Mariel-Majana

R4: Iglesia de Artemisa

R5: Logia Evolución

R6: Terminal R-35

R7: Mausoleo a los Mártires

R8: Hotel Campoamor

R9: Biblioteca Ciro Redondo

R10: Casa de la Música

R11: Palacio de los Matrimonios 
R12: Cementerio de Artemisa

R13: Museo de Artemisa

R14: Galería Angerona

R15: Boulevard de Artemisa

R16: Parque de la AHS

R17: Parque de la Edad de Oro

R18: Parque de la Iglesia

R19: Plaza Cultural de Artemisa

R20: Pupila Insomne

Con el objetivo de caracterizar cada recurso se realizó el inventario de los mismos, llegando a conformar diferentes grupos en dependencia de su clasificación con respecto al espacio y con respecto a la forma. Para llegar a esta clasificación fue de gran ayuda las fichas de recursos previamente elaboradas.

\section{Clasificación respecto al espacio:}

- Recurso natural y paisajístico: R1, R2.

- Recursos histórico monumental, técnicos, etnológicos y artísticos: R3, R4, R5, R6, R7, R8, R9, R11, R12, R13, R14, R15, R16, R17, R18, R19.

- Recursos artesanos y gastronómicos: R10, R20.

- Folclore, fiestas y acontecimientos programados: se han perdido muchas tradiciones a raíz del constante cambio de provincias. Solo existe una celebración por la fecha del 25 al 28 de abril, conocido como la semana de la cultura en Artemisa y dedicada a San Marcos pero que no se trata como tradición.

\section{Clasificación respecto a la forma:}

- Recursos actuales o potenciales: R1, R2, R3, R4, R5, R6, R7, R8, R9, R10, R11, R12, R13, R14, R15, R16, R17, R18, R19

- Recursos iniciativos singulares: R20 
Una vez clasificados los recursos se procedió a realizar las Tablas que sirvieron como herramientas, dentro de la observación científica, para la evaluación general de los recursos.

Tabla 1: Valoración del estado y conservación de los recursos, su carácter (disuasivo o atrayente), limitaciones para el uso y recomendaciones.

En las tablas elaboradas se pudo determinar el estado, uso actual y tendencial, así como la valoración de uso potencial de cada uno de los recursos, estableciendo para dicha valoración una calificación de (alto, medio y bajo). Estos valores representan su propio significado y se determinan en dependencia del estado de cada recurso en relación a cada categoría.

- Alto: su conservación es excelente, se realizan actividades en este o se puede desarrollar (óptimo).

- Medio: tiene un nivel de conservación bueno pero no óptimo, no se realizan muchas actividades en este o se pudiera desarrollar pero no dispone de muchos elementos que se lo permitan.

- Bajo: no presenta una buena conservación, no es frecuentado o es muy difícil lograr el desarrollo de este. Necesitan de una gran inversión para poder desarrollarlos.

La tabla, de forma general, arrojó resultados satisfactorios. No existe ningún atractivo que presente un bajo nivel de conservación. Con respecto al uso actual o tendencial, existen recursos/atractivos que presentan una calificación baja pero sin embargo, presentan un excelente nivel de conservación y pueden ser desarrollados para lograr un incremento de su uso. Debido a esto, las calificaciones bajas no afectan la evaluación de los recursos. Cuando evaluamos las potencialidades de los recursos, pudimos percatarnos de que no existen niveles bajo en relación al desarrollo de los mismos y por lo tanto, todos, unos más que otros, pueden ser desarrollados para garantizar y potenciar su uso.

En esta tabla también agregamos la evaluación de las limitaciones de uso, estableciéndose como notas evaluativas (si- presenta limitaciones, no- no presenta limitaciones). Los recursos que presentan limitaciones, se evaluaron en dependencia de su valor histórico cultural y por lo tanto se llegó a la conclusión de que era necesario establecer normas a la hora de interactuar con estos.

Hablando del carácter disuasivo o atrayente (Tabla 2), la totalidad de los recursos son atrayentes exceptuando el Cementerio del municipio, que en algunos casos puede actuar como disuasivo. Posterior y dependiendo de estas clasificaciones, se propusieron recomendaciones que fueron encaminadas a la preservación del recurso y el aumento de su atractividad. A modo general, la valoración de los recursos fue excelente. 
Tabla 3: Definición del valor de los recursos (unicidad, intensidad, geodiversidad).

La tabla define la valoración en relación a la unicidad (originalidad), intensidad (impacto) y geodiversidad (diversidad en su composición) de los recursos donde se dieron valores de 0 a 3, siendo 0 la no presentación de alguna categoría, 3 la presentación absoluta de la categoría por parte del recurso y 1 y 2 valores intermedios.

Esta tabla permitió realizar un promedio para cada recurso en relación a la presencia de las tres categorías de manejo y gracias a esto pudimos inferir que R6 es el recurso con más bajo promedio y por lo tanto su valor se encuentra amenazado. Haciendo referencia a las categorías de manejo, la geodiversidad, es decir la diversidad en su composición, es la categoría con más problema, no siendo así en relación a la intensidad y la unicidad que aunque no sean los valores menores, no están muy lejos del valor de geodiversidad por lo tanto, pudieran presentar problemas. Dentro de los recursos con mayor valor se encuentran: R1, R2, R3, R5 y R7, presentando estos los promedios más grandes.

Tabla 4: Valoración del uso funcional (según propuestas de actividades turístico recreativas y modalidades a desarrollar)

En la tabla se establece una evaluación de la relación directa entre los recursos/atractivos y las actividades y modalidades turísticas a proponer, es decir, se da una evaluación de 0 a 3 en la cual se puede inferir si el recurso está relacionado o puede vincularse con la actividad o modalidad turística. De esta manera se pudo valorar también la funcionalidad o no de la propuesta. Los valores representan: 0 no se relaciona, 1 puede tener algún tipo de relación, 2 tienen puntos o elementos en común y 3 son dependientes una de otra.

Luego de la realización de la tabla, se pudieron llegar a varias conclusiones, dentro de estas:

- La modalidad que más se puede desarrollar debido a que presenta un mayor número de recursos asociados a la misma, es el Turismo Cultural.

- Por último, los recursos más completos, valorando esta definición en dependencia a su importancia en relación a las actividades o modalidades con las que se relaciona o interactúan, son: R1, R2, R3, R4 Y R5.

Tabla 5: Evaluación turística de los recursos.

En esta última tabla se analizaron elementos relacionados con el turismo y se le dieron valores del 0 al 3, siendo 0 nulo y 3 el valor máximo. Dentro de los elementos evaluados están:

Evaluación estética: El estudio de las bellezas naturales que poseen los paisajes, incluye los aspectos siguientes: presencia y abundancia de elementos atrayentes, armonía de 
formas, gama de colores, grado de exotismo, posibilidades de observación de panoramas, contraste de formas del relieve, fondo de colores y sonidos, profundidad de la vista, alternancia de espacios abiertos y cerrados, etcétera.

Evaluación funcional: Analiza los diversos tipos de actividades turísticas recreativas que se pueden desarrollar en el área, partiendo de las características del territorio, de la necesidad de evitar cualquier degradación de los recursos y de los intereses recreativos de los clientes potenciales.

Evaluación conservacionista: Es el análisis de los valores naturales y culturales existentes, en cuanto a su relevancia natural y ecológica, su grado de exclusividad y necesidad de conservación.

Evaluación higiénico-sanitaria: Evalúa la calidad higiénico-sanitaria de las áreas, que pueden afectar los indicadores de confort de los clientes potenciales, teniendo en cuenta cualquier agente contaminante, la calidad de las aguas, el impacto ambiental y otros aspectos a considerar.

Evaluación técnico-económica: Estudia las posibilidades ingenieriles de realizar diferentes obras infraestructurales y su factibilidad económica, en este punto es necesaria la evaluación actual de las áreas.

Luego de todo el análisis realizado se pudo inferir que el recurso que mayor evaluación turística presenta es R8 (obteniendo 15 - máxima evaluación posible), seguido de R15, R10, R7 y R4. Por contraposición el recurso con menos valor turístico, dentro de la totalidad de recursos investigados, es R12 (Cementerio de Artemisa).

\section{Discusión.}

Una vez analizados los resultados que arrojo nuestra investigación, se procedió a desarrollar la fundamentación de las propuestas y la evaluación de impacto de las mismas (tanto ambiental como patrimonial). Finalmente quedaron como propuestas desarrolladas:

\section{Turismo de Naturaleza:}

Esta modalidad tendrá como principales recursos asociados el Cafetal Angerona y el Cafetal Buena Vista, ambos con un altísimo valor histórico-cultural y natural. Su enfoque estará guiado a la divulgación del patrimonio y la riqueza natural de los campos artemiseños. No presentará una estacionalidad definida y la accesibilidad para su desarrollo, es bastante favorable.

En relación a esta modalidad se diseñó la actividad: Haz tu café. Esta actividad comprenderá dos días y tendrá asociada a ella varias sub-actividades como, por ejemplo: 
la observación de un simulacro en los cafetales referente a la vida del siglo XIX en los mismos; la realización de trabajos relacionados con el cultivo, recolecta y procesamiento de café; excursiones en caballo y observaciones de ave. Esta actividad incluirá el almuerzo del primer día y el hospedaje en el Hotel La Moca de Las Terrazas, donde podrán presenciar de una hermosa vista.

\section{Turismo cultural:}

Esta modalidad tendrá como recursos asociados, los siguientes: R3, R5, R6, R7, R9, R10, R11, R12, R13, R14, R15, R16, R17, R18, R19, R20, todos estos se encuentran dentro del municipio, facilitando así la accesibilidad a la realización de esta modalidad. Cada uno de los recursos posee un elevadísimo valor histórico cultural por lo que el enfoque de esta modalidad está encaminado a la divulgación de la historia y la cultura del municipio, así como también al fortalecimiento del grado de concientización y sentido de pertenencia de sus pobladores.

En relación a esta modalidad, se diseñó la actividad: Ruta al Moncada. Esta actividad tendrá fundamentalmente los recursos: R3, R5, R6 y R7 como asociados y su objetivo principal será mediante un recorrido, ir contando la historia del municipio en relación a los principales lugares que han formado parte de ella. Por último, se realizará un tour a pie que enlazará lo que fueron los principales escenarios y lugares en los cuales los moncadistas artemiseños realizaban sus labores antes de partir hacia el Moncada. Culmina en el Mausoleo, lugar donde descansan los restos de los moncadistas.

\section{Turismo de eventos:}

Esta modalidad tendrá como recursos asociados: R3, R4, R7, R8, R9, R10, R11, R13 y $\mathrm{R} 14$. Su accesibilidad y estacionalidad iría en dependencia de las actividades asociadas que se realicen durante el año y su principal enfoque está encaminado a mantener viva la cultura del municipio y resaltar fechas importantes y conmemorativas para este.

En relación a esta modalidad se diseñó un evento que nombramos: Recordando a los mártires artemiseños. Dicho evento comprendería dos días y su pilar fundamental sería la realización de conferencias relacionadas con el desarrollo histórico-cultural del municipio. Estas conferencias se realizarían en espacios abiertos de libre acceso, facilitando la participación de los locales interesados, exceptuando el último día que sería la actividad de clausura en el Mausoleo. El evento incluiría el alojamiento en el Hotel Campoamor.

\section{Conclusiones:}

- Como resultado de la investigación podemos plantear que para aumentar el nivel de participación de Artemisa en el turismo nacional, sería factible la 
implementación del Turismo de Naturaleza, Turismo Cultural y Turismo de Evento, siendo estas modalidades las más posibles a realizar.

- Con el objetivo de poner en práctica o desarrollar las modalidades turísticas anteriormente mencionadas, se propusieron 3 actividades que están en estrecho vínculo con estas y que facilitarían la exposición de la cultura artemiseña así como también un aumento de los ingresos asociados al turismo. Dichas actividades son: Ruta al Moncada, Haz tu café y Recordando a los Mártires Artemiseños.

\section{Recomendaciones:}

- Es importante trabajar para contrarrestar los efectos negativos que se pudieran dar con la implementación de esta propuesta, dentro de estos: el sobreuso y daño del Patrimonio

\section{Referencias bibliográficas:}

Echarri, M., Salinas, E., \& Salinas, E. (2007). Apuntes de Geografía Turística de Cuba. La Habana.

Hicuba, W. (Noviembre de 2016). Las terrazas, turismo de naturaleza en la Sierra del Rosario. Recuperado el 20 de Noviembre de 2017, de hicuba.com

Jimenez, M., \& G., J. M. (1 de julio de 2008). El Municipio de Artemisa, Ciudades, Pueblos y Lugares de Cuba. Recuperado el 20 de Noviembre de 2017, de Guije.com.

ONEI. (2017). Anuario Estadístico de Artemisa. Artemisa.

Perelló Cabrera, J. L. (2005). Selección de textos sobre interpretación del patrimonio. La Habana: Centro de Estudios Turísticos.

Salinas Chávez, E. (2013). Geografía y Turismo. La Habana: Editorial Universitaria Félix Varela.

Suárez, D. (2 de Diciembre de 2017). Patrimonio de Artemisa. (G. Henández, \& H. Ronald, Entrevistadores)

Torres Moré, P. (s.f.). Gestión Tursística del Patrimonio Cultural. Selección de lecturas. La Habana.

Torres Moré, P. (s.f.). Técnicas de interpretación del Patrimonio Cultural. Selección de lecturas. La Habana.

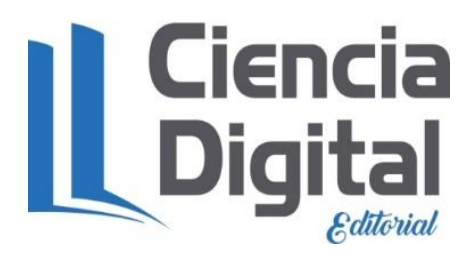




\section{PARA CITAR EL ARTÍCULO INDEXADO.}

Hernández Rodríguez, G. C. (2020). Propuestas de uso funcional para potenciar el turismo en el Municipio Artemisa como vía de desarrollo local. Explorador Digital, 4(3), 51-61. https://doi.org/10.33262/exploradordigital.v4i3.1279

\section{Ciencia \\ Ligital \\ Editorial}

El artículo que se publica es de exclusiva responsabilidad de los autores y no necesariamente reflejan el pensamiento de la Revista Explorador Digital.

El artículo queda en propiedad de la revista y, por tanto, su publicación parcial y/o total en otro medio tiene que ser autorizado por el director de la Revista Explorador Digital.
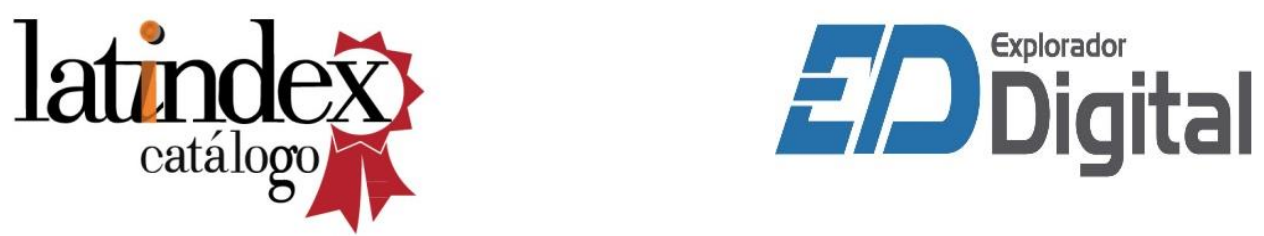